\title{
Entrapment of Gold Nanoparticles in Liposomes for Controlled Intracellular Self-assembly
}

\author{
Ananth S Pannala, Gennaro A Dichello, Prabal K Chatterjee
}

University of Brighton, $U K$

Background: Self-assembling nanomaterials (SANs) promise technological innovation at all stages of healthcare, encompassing fields of genomics, biosensors, immuno-analysis, drug delivery, detection, monitoring and treatment of diseases and infections. The generalised aim across these disciplines can be described as working towards the design of smart multifunctional nanosystems that interact, respond and provide treatment. To effectively exploit this approach, a high-level of behavioural understanding and control under biological conditions is required.

Methods: Molecular recognition and electrostatic attraction, two different strategies of gold nanoparticle self-assembly were studied. Corresponding nanoparticles were incorporated into PEGylated liposomes using a novel method. Two formulations were manufactured and characterised with gold and lipid concentrations determined using analytical and microscopy techniques. Toxicity evaluation between liposomal systems and corresponding gold nanoparticles was performed in-vitro on hamster lung fibroblasts (V79), employing MTT and LDH assays. Cellular uptake and selfassembly of nanoparticles was investigated using a combination of electron microscopy and elemental analysis.

Results: Both strategies facilitated spontaneous self-assembly of nanoparticles under aqueous conditions. However, within a biologically relevant medium considerable bio-complex formation occurred and only particles exploiting electrostatic interactions persisted to self-assemble. Nanoparticles were capable of being encapsulated within multilamellar liposomes by electrostatic exploiting interactions between oppositely charged components. The novel method resulted in variable internalised gold to lipid ratios, as a result of differing magnitudes of electrostatic attraction during preparation. Gold nanoparticles with cationic or anionic surfaces did not display cytotoxicity, although a significant difference in cytotoxicity was displayed as they underwent in-situ self-assembly. Liposomes with and without encapsulated gold nanoparticles exhibited significant dose-dependant cytotoxicity. Cellular internalisation of gold nanoparticles was evidenced within cellular vacuoles, although no confirmation of self-assembly was obtained.

Conclusions: Nanomaterial-biological interactions preceding the process of self-assembly can hinder activity and result in unpredictable outcomes. Individual SANs can be incorporated within traditional drug delivery systems, which could be further investigated towards controlling self-assembly activity. Toxicity studies demonstrate that a unique biological response could arise when nanomaterials self-assemble. Intracellular evaluation of SANs is inherently difficult and current techniques and approaches would benefit from further development to enable routine and reliable assessment of analogous nanosystems. 